\title{
2-碘酰基苯甲酸在有机合成中的研究与应用
}

\author{
张书瑜吴昊天 汤 峨* \\ (云南大学化学科学与工程学院 教育部自然资源药物化学重点实验室 昆明 650091)
}

\begin{abstract}
摘要 高价有机碘化合物的反应性质与过渡金属相似，其参与的反应具有反应条件较温和、选择性好、产率高及环境 友好等优点, 因而近年来关于高价有机碘试剂的研究受到广泛关注，在有机合成领域中获得了较多应用. 综述了近年 来高价有机碘试剂 2-碘酰基苯基酸(IBX)在有机合成中的研究及应用, 包括 IBX 在氧化差基、含氮化合物和含硫化合物, 在制备 $\alpha \beta$-不饱和羰基化合物和 $\alpha, \beta$-不饱和酯, 以及在不对称合成等方面的应用. 最后介绍了近期对 IBX 的改进.

关键词 2-碘酰基苯甲酸(IBX); 氧化; 有机合成; 高价有机碘
\end{abstract}

\section{Research and Application of 2-lodoxybenzoic Acid in Organic Synthesis}

\author{
Zhang, Shuyu Wu, Haotian Tang, E* \\ (Key Laboratory of Medicinal Chemistry for Natural Resources, Ministry of Education and Yunnan Province, \\ School of Chemical Science and Technology, Yunnan University, Kunming 650091)
}

\begin{abstract}
High-valent organoiodine reagents have many reaction characteristics similar to transition metals, and high-valent organoiodine-mediated reactions feature mild reaction conditions, good product selectivity, high yield and environmental friendliness. Therefore, they have been widely studied and applied in organic synthesis. The recent research progress of 2-iodoxybenzoic acid in organic synthesis is reviewed, including the oxidation of hydroxyl group, nitrogen-containing compounds and sulfur-containing compounds, the preparation of $\alpha, \beta$-unsaturated carbonyl compounds and esters, and the application in asymmetric synthesis. The recent improvement of IBX is also introduced.
\end{abstract}

Keywords 2-iodoxybenzoic acid (IBX); oxidation; organic synthesis; high-valent organoiodine

高价有机碘化学是有机化学学科的一个分支. 第一 个高价有机碘试剂是由 Willgerodt ${ }^{[1]}$ 于 1886 年合成的, 在之后的 120 余年里, 高价有机碘化学的发展较为缓慢, 直到 Dess-Martin ${ }^{[2]}$ 试剂 $[(1,1,1$-三乙酰氧基) $-1,1$-二氢1,2-苯碘酰-3(1H)-酮，DMP]出现后，因其优异的反应性 能, 使高价有机碘试剂在有机化学研究领域中获得了广 泛关注.

高价有机碘试剂中的碘有三价和五价两种价态. 在 其分子中, 碘的 $5 \mathrm{p}$ 轨道与配体的原子/分子轨道相互重 叠, 可形成新的三中心四电子或两中心四电子化学键. 这样的化学键较弱且高度极化, 具有较高的反应活性,
因而多种高价有机碘试剂被制备出来, 并在有机合成中 获得了广泛应用 ${ }^{[3]}$. 近年来，出现了许多关于高价有机 碘化合物参与的反应和应用研究的综述报道 ${ }^{[3]}$, 但关于 2-碘酰基苯甲酸(IBX)的综述报道则较少 ${ }^{[4]}$.

将 2-碘酰基苯基酸(IBX)与乙酸、乙酸酐混合加热 即可得到 Dess-Martin 试剂 $\mathrm{DMP}^{[2]}$ (Eq. 1). IBX 和 DMP 作为高价碘氧化剂, 具有反应性好及产率高等优点, 但 过去认为这两种高价碘试剂难溶于有机溶剂, 从而难以

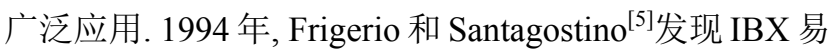
溶于二甲亚砜(DMSO). 自此, IBX 很快便成为了高价有 机碘化学新的研究热点. 相比于 DMP, IBX 更稳定, 可

\footnotetext{
* Corresponding author. E-mail: tange@ynu.edu.cn

Received July 9, 2020; revised August 11, 2020; published online September 15, 2020.

Project supported by the Program for Changjiang Scholars and Innovative Research Team in University (No. IRT13095), the Natural Science Foundation of China (No. 21762046), the Program for Yunnan Provincial Department of Science and Technology-Yunnan University "Double First-Class" Construction Joint Fund (No. 2018FY001), the Program for Scientifically and Technologically Innovative Research Teams in the Universities in Yunnan Province and the Program for the "Young Talent" in Yunnan University.

长江学者和创新团队发展计划(No. IRT13095)、国家自然科学基金(No. 21762046)、云南科技厅一云南大学 “双一流” 建设联合基金(No. 2018FY001)、 云南省高校科技创新团队支持计划及云南大学 “青年英才”培育计划资助项目.
} 
长期保存, 反应选择性也更好. 同时, IBX 还具有易于 制备和价格低廉等优点. 本文对近年来 IBX 在有机合成 领域中的研究进展及应用情况进行了综述.<smiles>O=C1O[Te](=O)(O)c2ccccc21</smiles>
$\underset{80{ }^{\circ} \mathrm{C}, 2 \mathrm{~h}, 91 \%}{\stackrel{\mathrm{Ac}_{2} \mathrm{O}, \mathrm{HOAc}, 100{ }^{\circ} \mathrm{C}}{\longrightarrow}}$ IBX<smiles>CC(=O)O[Te]1(OC(C)=O)(OC(C)=O)OC(=O)c2ccccc21</smiles>

\section{IBX 与羟基的反应}

\section{1 水溶液中 IBX 与醇的反应}

醇羟基氧化为羰基是有机化学中最基本的官能团 转化过程之一. 近年来, IBX 氧化醇、含氮及含硫有机化 合物的相关研究备受关注. IBX 可将伯醇和仲醇氧化为 相应的醛和酮, 且伯醇不会被继续氧化为羧酸. 此反应 条件温和, 产率高, 选择性好. 在过去 20 年间, IBX 在 DMSO 中的氧化反应屡见报道, 但其在水溶液中进行的 反应却并不常见.

在葫芦 [8]脲 $(\mathbf{Q}[8])$ 存在下, 芳基或烯丙基上的羟基 在水溶液中可被 IBX 选择性氧化为相应的醛 $4^{[6]}$. 先前 的研究主要是 IBX 在 DMSO 中或是在无溶剂条件下进 行的反应, 故这类可在水溶液中进行的反应在特定情况 下就显得十分有用(Eq. 2). Beaudry 等 ${ }^{[7]}$ 系统研究了 $\beta$-羟 基酮 5 被 IBX 氧化为 $\beta$-二酮 6 的反应(Eq. 3), 发现 IBX 是一种高效、易操作的氧化剂, 在毫克级和克级反应中 均呈现出较好的氧化性.
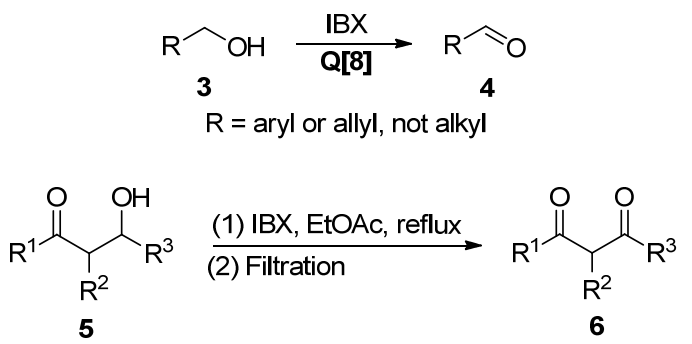

(3)

2015 年, 王伯良小组 ${ }^{[8]}$ 报道了一种水相中 IBX 氧化 苄醇 7 得到相应的酮 8 的反应. 他们在相转移催化剂四 丁基溴化铵(TBAB)存在下, 使用 IBX 在水溶液中将伯 醇选择性氧化为醛, 将仲醇选择性氧化为酮. 这是一种 反应条件温和、成本低且在水溶液中进行的催化氧化醇 成羰基化合物的绿色方法(Eq. 4).<smiles>CC(O)c1ccccc1</smiles>

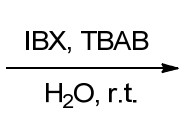
7<smiles>CC(=O)c1ccccc1</smiles>

8
(4)

GMPGS-2000 是由琥珀酸分别与亲水的聚乙二醇
单甲醚 (MPEG-2000) 和亲脂的 2-辛基十二烷-1-醇 (Guerbet 醇)脱水形成的非离子型两性聚合物, 其在水溶 液中能形成纳米胶束. 2014 年, 董伟等 ${ }^{[9]}$ 研究了 IBX 在 GMPGS-2000 纳米胶束存在下氧化醇的反应, 发现在水 溶液中及室温条件下，仲醇 9 可被 IBX 氧化为相应的酮 10, 产率高达 97\% (Eq. 5).

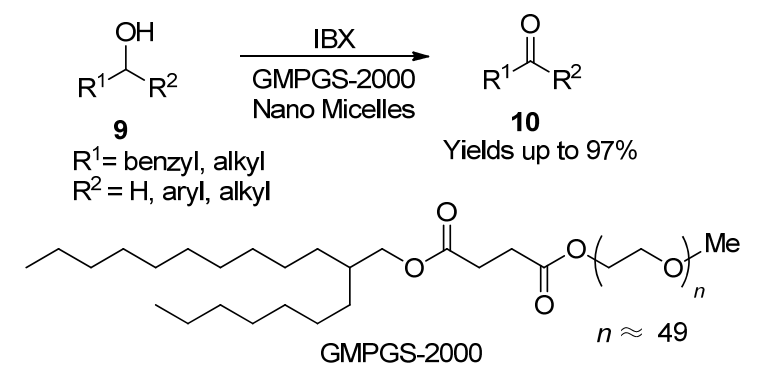

\subsection{IBX 与环氧化合物的反应}

环氧化合物是制备邻二羟基的常用前体，在氧化剂 IBX 存在下，可以将生成的羟基进一步氧化成羰基化合 物，羟基的保护和脱保护是多步有机合成中的常用策 略，在天然产物的全合成中具有重要意义. 2016 年, Kuma 和 Ahmed ${ }^{[10]}$ 发现 $\beta$-环糊精/IBX 体系能在水中对 $\alpha, \beta$-环氧乙烷基芳基酮 11 和 14 分子中的环氧乙烷基进 行氧化裂解及醇的氧化脱氢, 同时对四氢吡喃基(THP)/ 甲氧甲基(MOM)/乙酰基(Ac)/对甲苯磺酰基(Ts)保护的 着基进行温和、高效的脱保护，从而一锅法得到 3-羟基 1,2-二酮和 1,2,3-三酮化合物(Eqs. 6, 7), 产率可达到 $90 \%$ 以上. 在该反应中, $\alpha, \beta$-环氧乙烷基芳基酮和 IBX 分

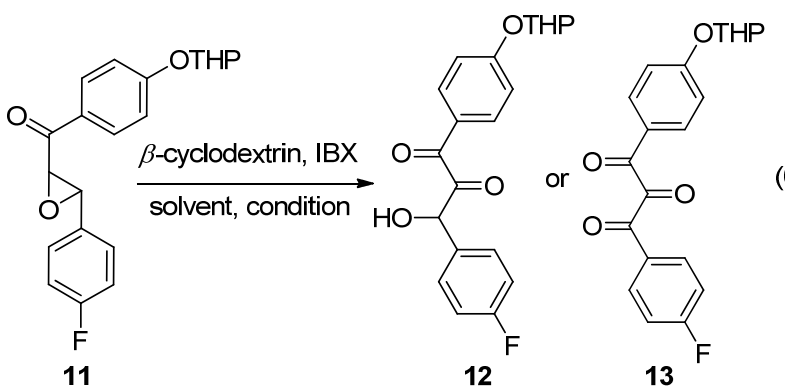

(6)

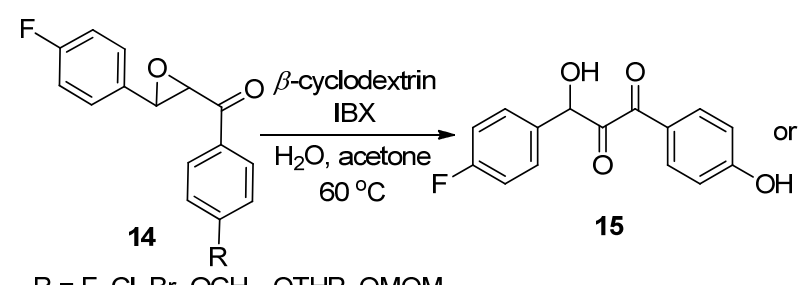

$\mathrm{R}=\mathrm{F}, \mathrm{Cl}, \mathrm{Br}, \mathrm{OCH}_{3}, \mathrm{OTHP}, \mathrm{OMOM}$<smiles>[R]c1ccc(C(=O)C(=O)C(O)c2ccc(F)cc2)cc1</smiles> 
子能同时进入 $\beta$-环糊精的空腔中，通过与 $\beta$-环糊精骨架 上的羟基形成氢键而得到活化; 同时, 由于 $\beta$-环糊精分 子骨架的特殊性使环氧化合物的开环反应的区域选择 性得到了有效控制(图 1).



图 1 IBX 氧化环氧化合物反应机理

Figure 1 Mechanism of the oxidation reaction of epoxy compounds by IBX

\subsection{IBX 氧化羟基的反应在近代有机合成中的应用}

IBX 能将醇着基控制性地氧化为羰基的优越性使 其备受合成工作者的青睐. 在近 10 年中，已有上百篇专 利或文章报道了 IBX 氧化着基的反应在多种天然产物 合成中的应用 ${ }^{[13-18]}$. 例如, 具有与紫杉醇类似的微管稳 定活性海洋天然产物鹅膏苷 $\mathrm{A}^{[11]}$ 、具有抗人类癌细胞增

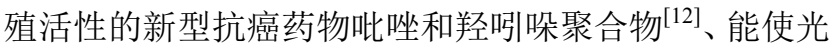
吸收能力得到增强的叶绿素衍生物 ${ }^{[14]}$ 、4-三氟乙酰基-3苯基亚砜晶体 ${ }^{[15]}$ 、菄皇式六环三萜化合物 ${ }^{[16]}$ 以及取代 1,2-环糖 ${ }^{[17]}$ 等天然产物的合成中都采用 IBX 作为优良氧 化剂，将醇氧化成相应的醛或酮.

\section{IBX 与含氮有机化合物的反应}

\subsection{IBX 氧化胺的反应}

将胺类化合物氧化为亚胺或其它种类的化合物是 有机合成中的常见过程，相关报道也有很多，但这些氧 化胺类化合物的方法或多或少存在着一定的缺陷，难以 在 $\mathrm{C}-\mathrm{N}$ 键形成的同时不产生其他有害物. 因而, 仍需 探索更加温和、方便的氧化胺的新方法.

2010 年 Telvekar 等 ${ }^{[19]}$ 在研究用 IBX 氧化苯甲酰肼 18 脱氢生成苯甲酸 20 或苯甲酸酯 21 的反应中发现, 当 反应体系中存在催化量的碱(如液氨)时, 脱氢后生成的 中间体会进一步释放氮气生成苯甲酰碳负离子，继而在 后处理中，苯甲酰碳负离子能捕捉质子形成苯甲醛 19 (Eq. 8). Telvekar 等的这一发现为 IBX 控制性氧化胺成 羰基提供了一个新的方法. 2017 年, 从航等 ${ }^{[20]}$ 实现了用
IBX 氧化二级苄胺化合物制备芳香甲醛. 该方法以氯仿 为溶剂, 在 $60{ }^{\circ} \mathrm{C}$ 左右的温度下进行, 后处理简单, 产 率最高可达 94\% (Eq. 9).

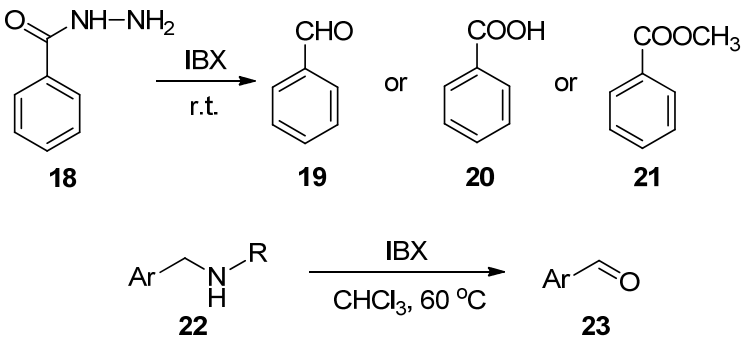

2011 年, Nageswar 等 ${ }^{[21]}$ 报道了一种在室温下, 借助 IBX 实现了水溶液中多取代的 1-芐基吡咯烷 24 和 $N$-芐 基脯氨酸类似物 26 的脱氢芳构化，合成出相应的取代 吡咯化合物 25 和 27 . 该反应同样是在 $\beta$-环糊精的帮助 下，利用吡咯烷化合物与 $\beta$-环糊精的主客体非共价键作 用，实现室温下的水相反应. 用 IBX 脱去杂环上的氢， 几乎不产生有害副产物，使之成为一种环境友好的 “绿 色”且经济的吡咯化合物的合成方法(Eqs. 10, 11).

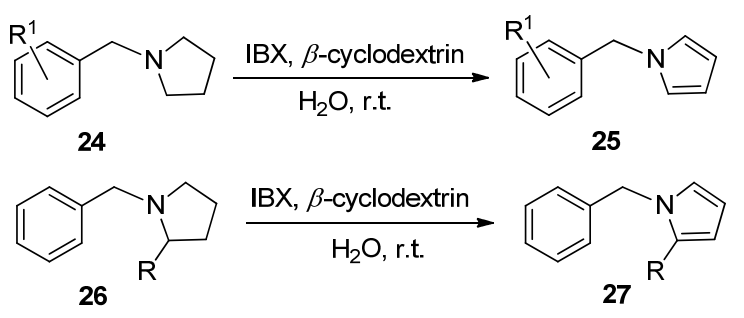

2016 年, Govidasamy 等 ${ }^{[22]}$ 研究了 IBX 存在下的具 有化学选择性的 2-氨基芳基酮 28 的串联反应. 该反应 包含三个连续的步骤，第一步是 2-氨基芳基酮苯环上的 着基官能化，第二步是将酚羟基氧化为醌 31, 第三步是 醌羰基与另一分子的氨基缩合生成亚胺基烯酮 29 (Scheme 1). 此外，该反应的副产物碘苯甲酸(IBA)可被 氧化为 IBX, 从而得到有效的回收利用(Eq. 12).

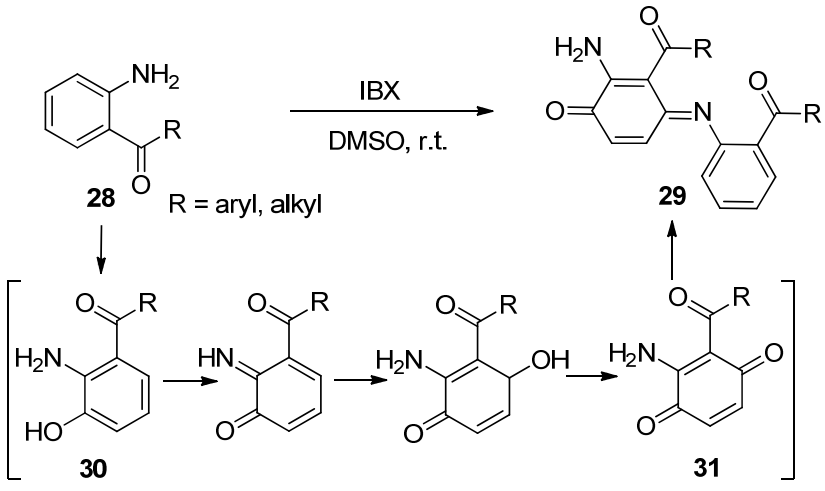

图式 1 IBX 参与的 2-氨基芳基酮 $\mathbf{2 8}$ 的串联反应 Scheme 1 IBX-mediated cascade reaction of 2-aminoaryl ketones $\mathbf{2 8}$ 
<smiles>O=C1OI(O)c2ccccc21</smiles>

IBA

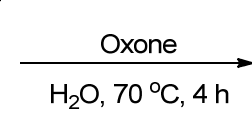<smiles>O=C1OI(=O)(O)c2ccccc21</smiles>

IBX
2015 年, Goti 等 ${ }^{[24]}$ 对 IBX 氧化着胺的反应进行了研 究(Eq. 13), 证明 IBX 是差弪胺的优良氧化剂, 且该方法高 效易操作. 两年后, Goti 等 ${ }^{[23]}$ 发现通过改变溶剂体系和 反应温度, 可以选择性地获得不同的产物. 在二氯甲烷 中, 室温搅拌反应 $2 \mathrm{~h}$, 差胺可被 IBX 氧化成硝酮化合 物 33, 这一反应产率高、区域选择性好. 如果用二甲亚 砜(DMSO)作溶剂, $45{ }^{\circ} \mathrm{C}$ 下反应则可以得到肜类化合物 36 (Scheme 2).

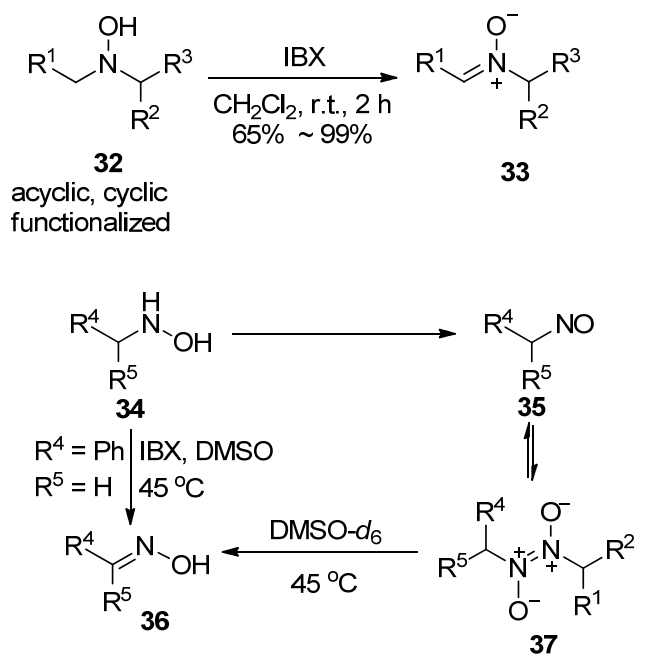

图式 2 IBX 氧化差弪胺成肜可能的反应机理

Scheme 2 Proposed mechanism of the oxidation reaction of hydroxylamines to oximes by IBX

\subsection{IBX 在含氮杂环化合物合成中的应用}

许多药物和具有生物活性的天然产物都属于含氮 杂环化合物. 因此, 含氮杂环化合物的合成与研究一直 是有机合成化学的重要课题. 在众多合成方法中, 过渡 金属参与的催化合成被认为是一种高效、高选择性的方 法. 然而，过渡金属催化法还存在底物适用范围有限、 反应条件苛刻(高温)及催化剂用量大等缺点, 使其在工 业上的应用受到很大限制.

Moorthy 课题组 ${ }^{[25]}$ 报道了在 DMSO 中, 用 IBX 将伯 醇 38 或 1 溴代烷氧化成醛, 继而与邻苯二胺 40 发生分 子间缩合制备出相应的苯并咪唑化合物 42. 尽管 IBX 氧化 $1^{\circ}$ 溴代烷成醛的反应温度要 $70{ }^{\circ} \mathrm{C}$, 但氧化和环合 两步反应一锅完成的合成策略仍是一种简便、快捷的好 方法(Scheme 3).

2018 年, Mal 等 ${ }^{[26]}$ 观察到当苯胺和 IBX 在无溶剂下 混合时，在最大接触条件下，立即发生了爆炸. 然而,

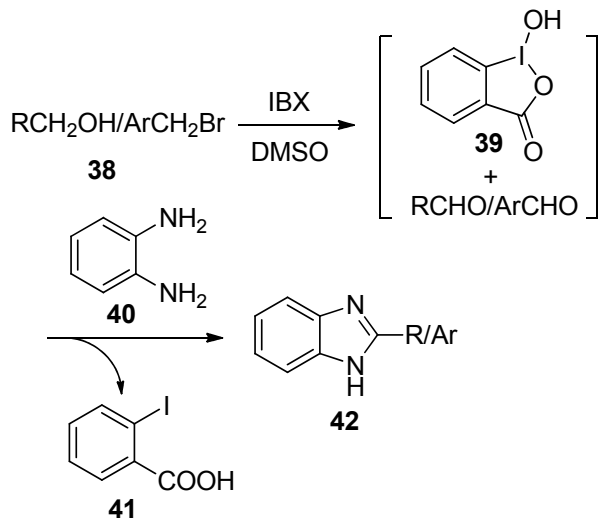

图式 3 苯并咪唑化合物 $\mathbf{4 2}$ 的合成

Scheme 3 Synthesis of benzimidazole compounds $\mathbf{4 2}$

当 2-氨基苯甲酰胺 43 和芳醛反应时，在相同条件下没 有爆炸. 因而，他们通过控制 IBX 的用量，以 2-氨基苯 甲酰胺为原料, 在无溶剂条件下成功合成出喹唑啉4(3H)-酮化合物 44, 反应的产率中等(Eq. 14).

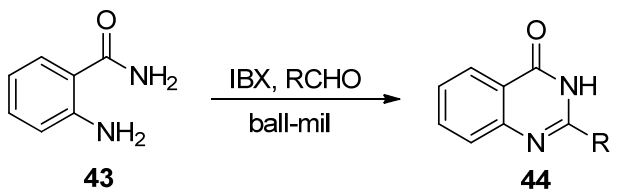

啞二唑在医药、农药、高分子和材料科学等领域都 是一种十分重要的分子骨架结构. 尽管已经报道了多种 制备噁二唑化合物的方法，但大多数方法都存在如反应 温度高、反应时间长、需强碱或强酸性条件及使用剧毒 或腐蚀性试剂等缺点. 2018 年, Subba Reddy 等 ${ }^{[27]}$ 报道了 IBX/KI 体系促进下, 2,5-二取代 1,3,4-腎二唑 46 的一锅 合成法. 在室温下, IBX 的作用包括把 $\mathrm{KI}$ 氧化成 $\mathrm{I}^{+}$离子 以及使二氢啞二唑氧化脱氢成啞二唑. 该方法具有反应 条件温和、试剂廉价和反应时间短等优点(Eq. 15).



2013 年, Bhanage 等 ${ }^{[28]}$ 则是利用 IBX 实现苯并噁唑 2 位 $\mathrm{C}-\mathrm{H}$ 的胺基官能化. 该反应具有简便、温和、反 应时间短及无需金属试剂等优点. 在该方法中, 苯并惡 唑 47 在胺的作用下开环, 形成胺基亚胺化合物 48, 苯 环上邻位的羟基对亚胺基进行亲核加成，形成苯并二氢 啞唑, 继而在 IBX 氧化下脱氢, 最终实现 2-胺基苯并噁 唑化合物的简便合成(Scheme 4).

喹诺酮类化合物具有抗菌、抗癌、抗艾滋病病毒 (HIV)、抗结核和抗疮等多种活性. 2014 年, Muraleedharan 等 ${ }^{[29]}$ 开发了一种喹诺酮、䒺啶酮和苯并萗啶酮 


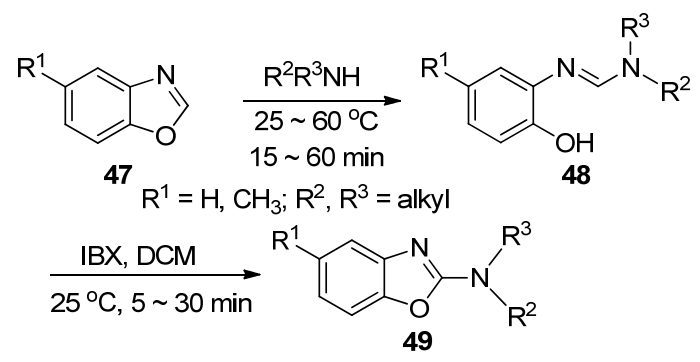

图式 4 2-胺基苯并噁唑化合物 49 的合成

Scheme 4 Synthesis of 2-aminobenzoxazole compounds 49

化合物的快速无金属试剂参与的合成方法(Scheme 5). 这个两步串联合成法, 是从 Baylis-Hillman 加合物 $\mathbf{5 0}$ 出 发, 先进行胺的迈克尔加成反应, 继而胺与缺电子卤代 芳烃进行 $\mathrm{S}_{\mathrm{N}} \mathrm{Ar}$ 环化反应，最后用 IBX 氧化羟基成羰基， 最终获得喹诺酮类、菜啶酮类和苯并荎啶酮类化合物 52. 该方法底物适用性广，无需金属试剂参与，产率高.<smiles>[R]C(=C)C(O)c1cc([R])c([R])c([Y])c1C([R])C(N)=O</smiles>



$\mathrm{R}^{1}, \mathrm{R}^{2}=\mathrm{H}$ or part of aromatic ring $\mathrm{X}=\mathrm{Cl}, \mathrm{Br}$ $\mathrm{R}^{3}=\mathrm{COOMe}, \mathrm{CN}$

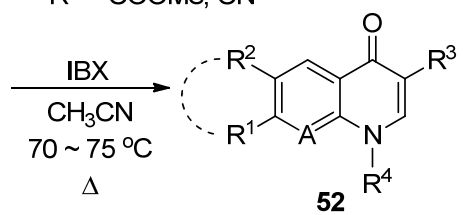

图式 5 喹诺酮、萗啶酮和苯并菜啶酮化合物 52 的合成 Scheme 5 Synthesis of quinolone, naphthyridone and benzonaphthyridone compounds $\mathbf{5 2}$
2019 年, Kanizsai 等 ${ }^{[30]}$ 报道了一种从 Mannich 反应 的产物 55 出发, 在 IBX/ $N$-碘代琥珀酰亚胺(NIS)促进下 发生氧化环合反应, 构建咪唑并 [1,2- $a$ 吡啶和咪唑并 [1,2- $a]$ 嘧啶分子骨架的简便方法. 在该方法中, NIS 诱导 化合物 55 分子中 1,3 -二羰基的 $\alpha-\mathrm{C}$ 发生碘代生成中间 体 56, 继而 IBX 氧化中间体 56 的胺基成亚胺，再通过 分子内亲核取代反应形成新的 $\mathrm{C}-\mathrm{N}$ 键，最后在 $\mathrm{I}^{-}$离子 协助下中间体 61 发生逆 Claisen-Schmidt 重排反应，脱 去酰基，从而高效地构建出咪唑并 [1,2- $a]$-吡啶和咪唑 并 $[1,2-a]$ 嘧啶分子骨架 56 (Scheme 7). 这一方法成功替 代了原有的过渡金属催化法，反应产率可达 $93 \%$ (Scheme 6).

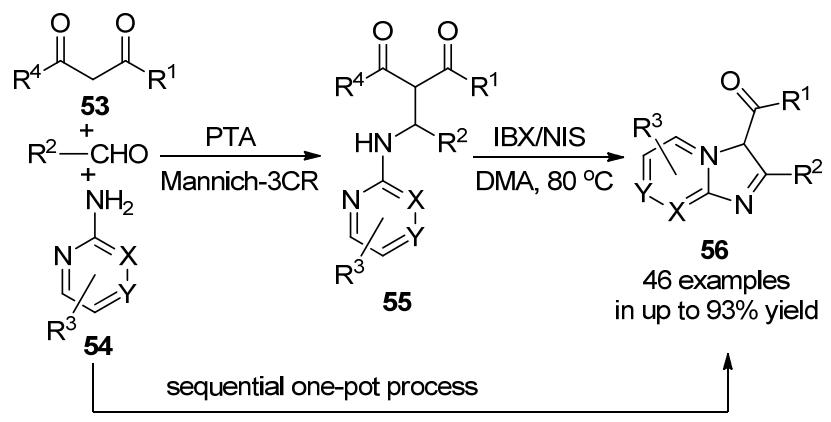

图式 6 IBX/NIS 促进氧化环化

Scheme 6 IBX/NIS-promoted oxidation cyclization

\subsection{IBX 在含氮化合物中的其它应用}

研究者们曾认为无法利用 1,3-二羰基化合物生成叠 氮化合物，但利用单质碘与叠氮盐却可以生成 $\alpha$-叠氮基 取代的 1,3-二羰基化合物，而用氧化剂与磑盐原位生

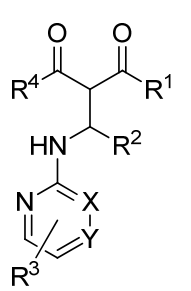

55<smiles>CN1C(=O)C(C)(C)C(C)(CC2CCCC2)C1=O</smiles>

$\mathrm{NHS}$<smiles></smiles>

58

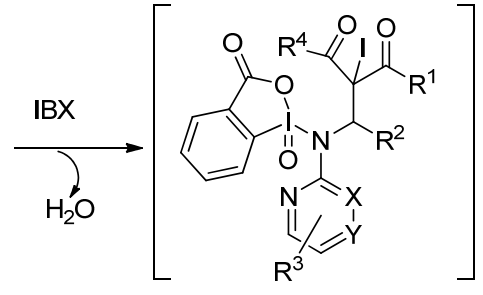<smiles>[13CH2]CC[I-]1[IH]c2ccccc2C1=O</smiles>

$\mathrm{OH}$

BA

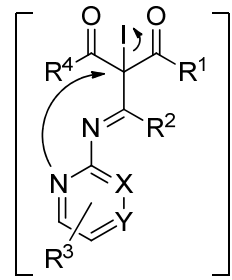

60

图式 7 可能的氧化环化机理

Scheme 7 Plausible mechanism of the oxidation cyclization 
成单质碘进行反应, 可以只使用催化量的碘盐即可完成 反应，大大提高了反应的效率. 相比传统氧化剂，IBX 显现出了更加优越的官能团耐受性和更广泛的底物适 用范围. 2012 年, Kirsch 等 ${ }^{[31]}$ 采用对羟基不反应的 IBX 磺酸钾盐 $\left(\mathrm{IBX}-\mathrm{SO}_{3} \mathrm{~K}\right)$ 作为碘化钠的温和氧化剂, 只需用 0.2 equiv. 的碘化钠, 即可原位生成单质碘与叠氮化钠和 1,3-二羰基化合物进行反应，成功开发了一种水相中制 备 $\alpha$-叠氮基取代的 1,3-二羰基化合物 63 的简便方法(Eq. 16).

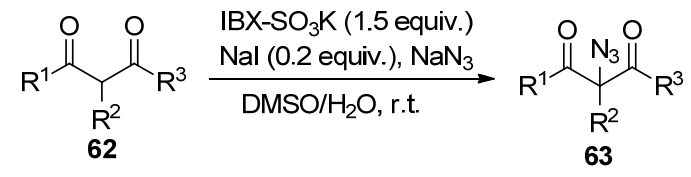

$N$-亚硝胺是致癌物, 在动物实验中常被用作诱变 剂. $N$-亚硝胺还是潜在的一氧化氮供体, 因而具有很好 的生物活性. 2012 年, Hou 等 ${ }^{[32]}$ 将仲胺或叔胺 64 在硝基 甲烷存在下, 用 IBX 作氧化剂, 在季铵盐的协助下实现 了脂肪族或芳香族 $N, N$-二取代亚硝胺 65 的合成(Eq. 17). 但是, 该反应的产率受季铵盐的影响较大, 其卤离 子的反应活性顺序为 $\mathrm{F}^{-}>\mathrm{Cl}^{-}>\mathrm{Br}^{-} \approx \mathrm{I}^{-}$, 当使用 2 equiv. 的四丁基氟化铵时, 产率可达 93\%. Hou 认为反应 可能分 3 步进行: 第一步, IBX 与季铵盐和硝基甲烷反 应先生成亚硝酸; 第二步, 三级胺 64 的 $N$-亚硝基化及 氧化脱烷基化反应生成二级胺; 第三步, 二级胺的亚硝 基化反应生成最终产物 65.

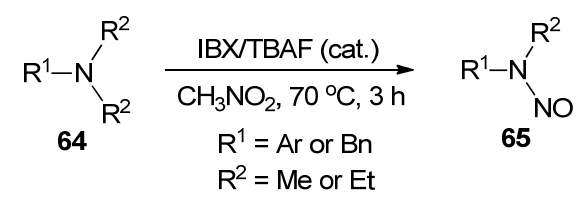

2018 年, 张志国等 ${ }^{[33]}$ 报道了 $N$-芳基酰胺分子中活 性低的芳基 $\mathrm{C}-\mathrm{N}$ 键的选择性裂解反应(Eq. 18). 研究发 现，这类 IBX 促进下高选择性的 $\mathrm{C}-\mathrm{N}$ 键裂解反应，可 在温和且无金属试剂存在的条件 [六氟异丙醇 (HFIP) $/ \mathrm{H}_{2} \mathrm{O}, 25^{\circ} \mathrm{C}$ ]下顺利进行. 在反应活性更高的酰 胺键保持不变的情况下，二级酰胺上反应性低的芳基 $\mathrm{C}$ $-\mathrm{N}$ 键优先得到氧化裂解. 该反应有效扩展了制备一级 酰胺的途径, 并可制备出采用传统氨解法和水解法无法 得到的酰胺化合物.

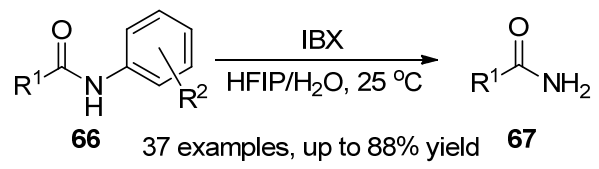

2019 年, Srivastava 等 ${ }^{[34]}$ 将 IBX 直接用于异腈对环
状二级胺 68 的氧化加成反应中，成功合成了 1-位酰胺 基取代的色氨酸和 1,2,3,4-四氢异喹啉衍生物 69 (Eq. 19). 在这一反应中 IBX 既是氧化剂, 又是 Lewis 酸催化 剂, 活化了氧化产物亚胺成亚胺盐中间体，提高了亚胺 碳的亲电性，从而活化了异腈对亚胺盐中间体的亲核加 成反应。该方法原子经济性高，底物范围广泛，操作简 单，条件温和，不失为一种高效合成 1-位含氮杂环基取 代的酰胺化合物的通用方法.



8-氨基喹啉(AQ)是一种广泛应用于过渡金属催化 下 $\mathrm{C}-\mathrm{H}$ 官能化反应中过渡金属离子的双齿配体. 2019 年, 张志国 ${ }^{[35]}$ 用化学计量的 IBX 作为氧化剂, 实现了 8氨基喹啉衍生物 70 分子中 $\mathrm{C}-\mathrm{N}$ 间的氧化裂解. 酰胺化 合物 71 在醋酸存在下水解就可以方便地获得 $\alpha$-氨基酸 衍生物 72. 这一反应在氧化剂单过硫酸氢钾(oxone)存 在时，只需用催化量的 IBX 即可顺利进行(Scheme 8). 该方法为 $\alpha$-氨基酸衍生物的合成提供了新的思路.
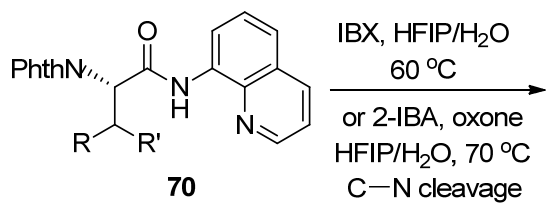<smiles>[R]C([R])[C@H](Nc1ccccc1)C(N)=O</smiles><smiles></smiles>

图式 $8 \alpha$-氨基酸衍生物的合成 Scheme 8 Synthesis of $\alpha$-amino acid derivatives

\section{IBX 与含硫有机化合物的反应}

亚砜是有机合成中一类非常有用的化合物，可通过 氧化硫醚获得，并可通过一系列反应转化为多种含硫有 机化合物. 因此亚砜在含硫药物和含硫天然产物的合成 中具有重要作用.

因为硫醚很容易被氧化成亚砜和砜，所以在将硫醚 氧化为亚砜时，通常需严格控制实验条件才能减少副产 物砜的生成. Akamanchi 等 ${ }^{[36]}$ 利用 IBX 在相转移催化剂 四乙基溴化铵(TEAB)存在下, 不仅将硫醚定量地氧化 为亚砜, 而且该反应没有产生常见副产物砜(Eq. 20). 若 不添加 TEAB, 反应速率较慢. 2019 年, Marques 等 ${ }^{[37]}$ 也 报道了采用类似高价碘试剂进行无金属试剂参与的磺 酰化反应. 


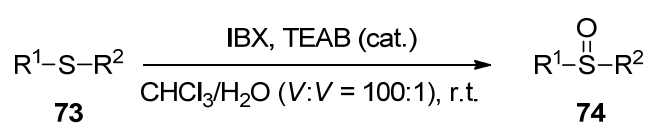

Moorthy 等 ${ }^{[38]}$ 分别制备了 IBX、3,5-二甲基取代的 IBX 衍生物(DiMe-IBX)和 2,3,4,5-四甲基取代 IBX 衍生 物(TetMe-IBX), 并用密度泛函理论计算优化了 IBX 及 DiMe-IBX 和 TetMe-IBX 的结构, 考察了三个氧化剂在 将伯醇氧化成醛的反应. 经过理论计算发现, TetMeIBX 中的四个甲基会使其与醇经配体交换反应形成的 高价碘中间体发生结构扭曲, 从而大大降低了相应的活 化能, 而这一步恰是氧化反应的决速步. 并且相互处于 邻位的甲基使分子结构在空间上形成了接力扭曲, 从而 使其在普通有机溶剂中的溶解性显著提高. 因此, TetMe-IBX 可以让醇和硫化物在室温下和普通有机溶剂中 也能顺利进行氧化反应(Eqs. 21 23).<smiles>Cc1c(C)c(C(=O)O)c(I)c(C(=O)O)c1C</smiles>

75<smiles>Cc1c(C)c(C)c2c(c1C)C(=O)OP2(=O)O</smiles>

76

$$
\begin{aligned}
& \begin{array}{ccc}
\mathrm{R}^{1}-\mathrm{S}-\mathrm{R}^{2} & \text { TetMe-IBX } & \stackrel{\mathrm{O}}{\mathrm{II}} \\
\mathbf{7 7} & \mathrm{DCM} \text {, r.t., } 10 \mathrm{Rin} \sim 2 \mathrm{~h} & \mathbf{7 8}-\mathrm{R}^{2} \\
& & \mathbf{7 8}
\end{array}
\end{aligned}
$$

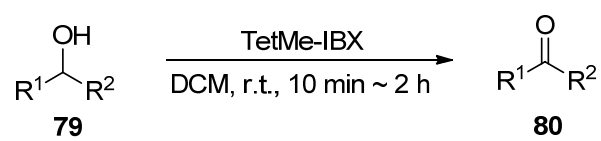

\section{IBX 制备 $\boldsymbol{\alpha}, \boldsymbol{\beta}$-不饱和羰基化合物}

$\alpha, \beta$-不饱和羰基化合物是一类重要的有机合成中间 体, 被广泛应用于天然产物、功能材料和药物分子的合 成中. 目前, 这类化合物的制备方法有 Aldol 缩合反应、 Wittig 反应、Hornor-Wadsworth-Emmons 反应、MeyerSchuster 重排等. 2013 年, IBX 引起了 Iida 等 ${ }^{[39]}$ 的注意, 他们在从甲酰氧基-5 $\beta$-胆甾醇-3-酮 81 合成甲酰氧基-4胆甾醇-3-酮 82 的研究中, 发现 IBX 是优良的氧化脱氢 试剂(Eq. 24).

2015 年, 曾步兵等 ${ }^{[40]}$ 用 IBX 将四氢荎酮 83 氧化成 萗醌. 在 DMSO 溶剂中, 直接用 IBX 氧化四氢萗酮 83 可得到 1,2-荎醌化合物 84. 当在单过硫酸氢钾(Oxone) 和相转移催化剂四丁基溴化铵( $\mathrm{TBAB}$ )存在下, 硝基甲 烷作溶剂时, IBX 则将四氢荎酮 83 氧化成 1,4-萗醌化合 物 85 (Scheme 9).

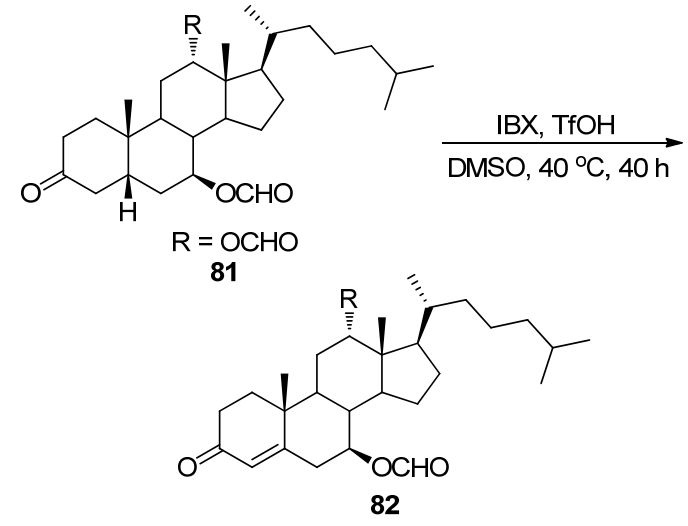



图式 9 IBX 的选择性氧化四氢菜酮

Scheme 9 Selective oxidation of 3,4-dihydronaphthalen-1(2H)one with IBX

2018 年, Iwabuchi 等 ${ }^{[41]}$ 发现 IBX 可催化过氧化物脱 水成羰基. 他们以 IBX 为催化剂, 研究了烯丙醇氢过氧 化物 86 在对甲苯磺酸和水存在下，脱水生成 $\alpha, \beta$-不饱和 羰基化合物 87 的反应(Eq. 25). 烯丙基过氧化醇可以由 烯烃与单线态氧反应制备, 因而这一反应提供了一种由 烯烃制备 $\alpha, \beta$-不饱和羰基化合物的简便方法. 加之反应 的副产物仅为 $\mathrm{H}_{2} \mathrm{O}$, 所以这一方法也体现了绿色合成的 特点.

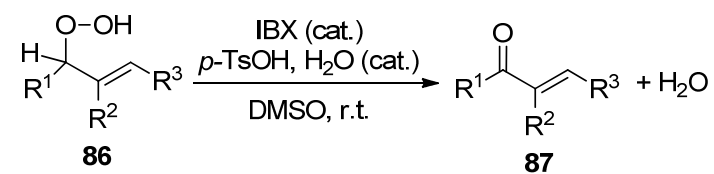

\section{IBX 在制备酯类化合物中的应用}

\subsection{IBX 在制备 $\beta$-氧代羧酸酯中的应用}

$\beta$-氧代羧酸酯不仅是生物活性化合物的重要骨架, 而且还是重要的有机合成中间体. 氧化 $\beta$-差弪基羧酸酯是 合成 $\beta$-氧代羧酸酯的最经典、最直接的方法之一. 2013 年, Rodrigues 等 ${ }^{[42]}$ 发现使用 IBX 氧化 Morita-BaylisHillman 加成产物 $\mathbf{8 8}$ 可制备出亚甲基酮酯化合物 $\mathbf{8 9}$, 反 应产率大于 $90 \%$ (Eq. 26).

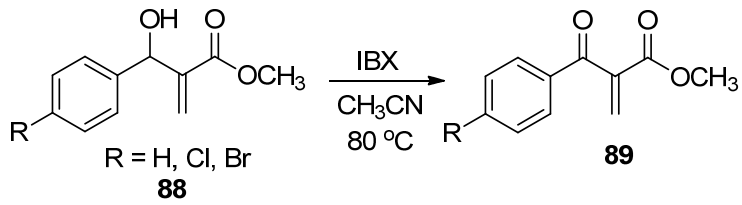


2015 年, Ilangovan 等 ${ }^{[43]}$ 开发了一种单质碘催化下, $\mathrm{IBX}$ 参与的 $\mathrm{sp} 、 \mathrm{sp}^{2}$ 和 $\mathrm{sp}^{3}$ 碳 $\mathrm{C}-\mathrm{H}$ 胺基化反应. 单质碘 催化下 2'-胺基苯乙炔 90、2'-胺基苯乙烯 91 和 $2^{\prime}$-氨基- $\beta$ 酮酯 92 的串联反应均可以制备吲哚啉-2,3-二酮化合物 93. 不论通过哪条路线制备吲哚啉-2,3-二酮, 反应均可 在空气中、非金属试剂参与的条件下进行 (Scheme 10).

2018 年, Sekar 等 ${ }^{[44]}$ 开发了一种新的 $\alpha$-羟基酮和醇 的氧化酯化串联反应制备 $\alpha$-酮酯 96 的新方法. 他们发 现在所有脂肪醇都能发生这种串联反应, 生成相应的 $\alpha-$ 酮酯. 当减少 IBX 的量的时, $\alpha$-酮酯的产量会降低. 没 有 IBX 存在时, 不产生 $\alpha$-酮酯; 同样, 如果没有 $\mathrm{I}_{2}$ 存在, 也只有微量的 $\alpha$-酮酯生成(Eq. 27).

为深入了解 $\alpha$-酩酯的形成机制, 他们在实验和文献 的基础上提出了一条可能的反应机理, 即 $\alpha$-差基酮 94 先被 IBX 氧化成 2-氧代-2-苯乙醛中间体 97, 继而在单 质碘和碱存在下与茮醇发生亲核加成, 脱去碘化氢得到 2-氧代-2-苯基醋酸芐酯 96 (Scheme 11).

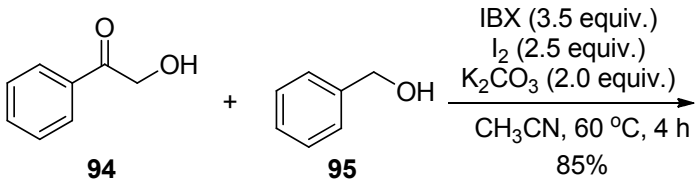<smiles>O=C(OCc1ccccc1)C(=O)c1ccccc1</smiles>

\subsection{IBX 在制备 $\beta$-碘代酯中的应用}

2017 年, Shaw 等 ${ }^{[45}$ 通过使用醋酸碘苯(BAIB)或 IBX, 在单质碘存在下, 从同一个三醋酸二氢吡喃酯 101 出发, 在室温下就能高度选择性地合成 3,4,6-三醋 酸-1-苯甲酸葡萄糖酯 $\mathbf{1 0 3}$ 和 1,3,4,6-四醋酸-2-硔代葡萄 糖酯 102, 而且两种 2-碘代葡萄糖酯产物均具有很高的 $\alpha$-型立体选择性(Scheme 12).

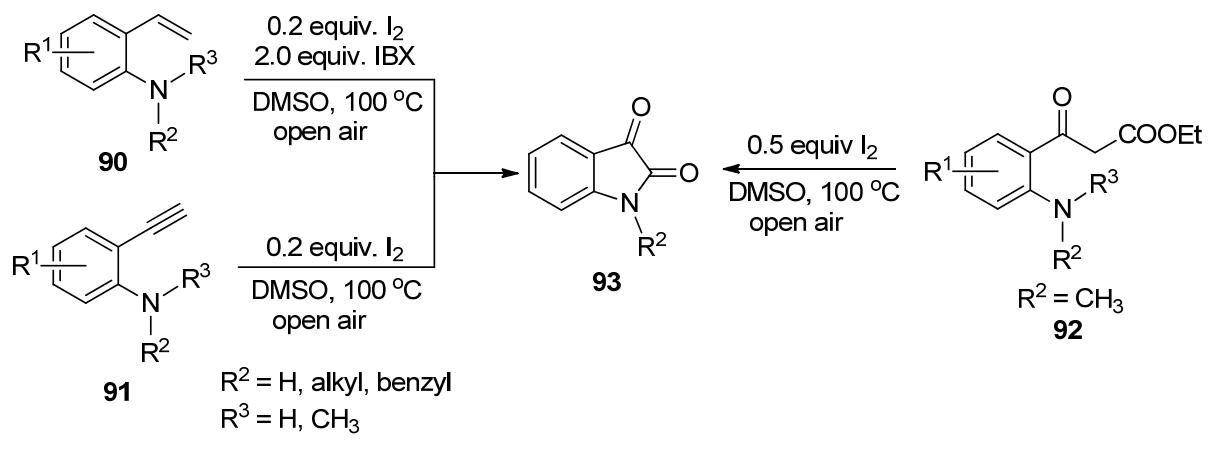

图式 10 吲哚啉 2,3-二酮化合物 93 的合成

Scheme 10 Synthesis of indoline-2,3-dione 93

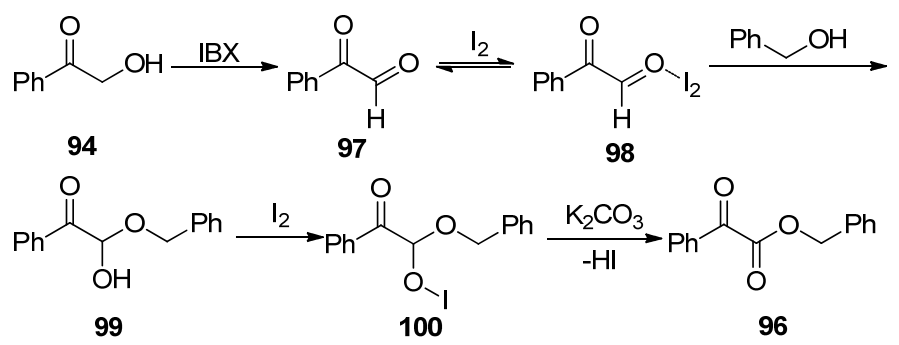

图式 11 IBX- $\mathrm{I}_{2}$ 氧化 $\alpha$-着基酮的可能机理

Scheme 11 A possible mechanism of the oxidation reaction of $\alpha$-hydroxyketone by IBX-I $\mathrm{I}_{2}$



图式 12 反式-2-脱氧-2-碘糖基乙酸酯和邻碘苯甲酸酯的选择性合成

Scheme 12 Selective synthesis of trans-2-deoxy-2-iodoglycosylacetates and $O$-iodobenzoates 


\section{IBX 在不对称合成中的应用}

不对称合成是指向反应物中引入一个或多个手性 原子的化学反应, 在药物合成和天然产物全合成中都有 十分重要的地位. 2014 年, Kim 等 ${ }^{[46]}$ 提出了一种具有对 映选择性的有机小分子催化下，2-胺基苯丙醛 104 分子 内环合制备四氢喹啉化合物 105 的方法. 这一方法用 IBX 作为氧化剂, 经过二级手性胺与醛基脱水缩合、 IBX 氧化烯胺中间体、1,5-氢迁移及 $e n d o$-型分子内环一 系列串联反应, 最终以中等产量和较好的对映选择性合 成出目标化合物(Eq. 28).

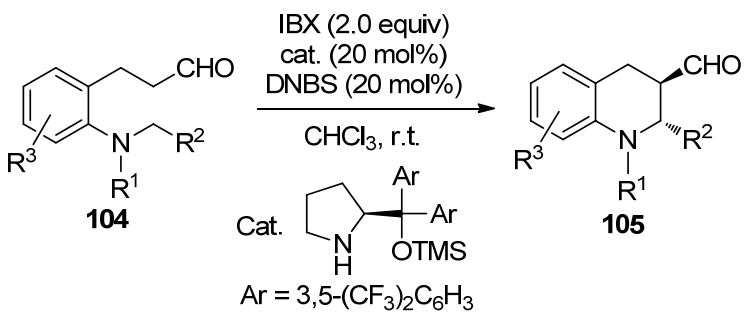

\section{IBX 在有机合成中的其他应用}

\subsection{IBX 参与下黄酮化合物的修饰和合成}

黄酮化合物有着 2-苯基色原酮的分子骨架，是一类 广泛存在于自然界中并具有多种生物活性的化合物. 2010 年, Bernini 等 ${ }^{[47]}$ 发现 IBX 可以使差基黄烷酮 106 的 $\mathrm{A}$ 环羟基官能化, 而且可以将甲氧基取代的黄烷酮 108 氧化脱氢成黄酮化合物 109 , 从而开发出一条制备
具有抗癌活性的儿茶酚衍生物高效方法. 同时, Bernini 等 ${ }^{[47]}$ 用制备出的聚苯乙烯负载的 IBX 代替小分子 IBX, 有效解决了高价碘试剂的回收和再利用的问题(Scheme 13).

2017 年, Desai 等 ${ }^{[48]}$ 用邻羟基查尔酮 110 为原料，通 过 IBX 促进下羟基对碳-碳双键的分子内亲电环合及氧 化脱氢反应，一步合成出 15 个黄酮化合物 111. 这一方 法的产率高, 没有金属试剂参与, 符合绿色化学的要求 (Eq. 29).

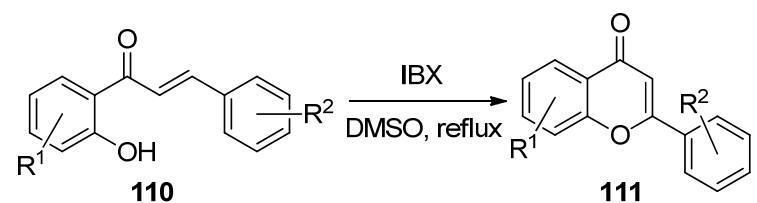

\subsection{IBX 在脱芳构化中的应用}

许多天然产物及合成化合物均具有芳环结构. 由于 芳香族化合物结构的特殊性，其去芳构化一直是有机合 成的一个巨大挑战. 吡喃氮酮是近年来被广泛研究的一 种具有细胞毒性的天然产物，而其二氢吡喃氮酮的合成 方法却鲜有报道. 2011 年, Franck 等 ${ }^{[49]}$ 在合成二氢吡喃 氮酮 114/115 骨架时，发现 IBX 可使芳香化合物去芳构 化(Scheme 14). 虽然反应机理尚不清楚，但可以肯定的 是高价碘试剂 IBX 是反应顺利进行的关键.

\subsection{IBX 在调整碳链长度中的应用}

有机合成中, 时常需要调整碳链的长度. 然而, 现<smiles>O=C1CC(c2ccccc2)Oc2ccc(O)cc21</smiles>

106 (a) IBX or IBX polystyrene $\underset{\mathrm{DMSO}, 25^{\circ} \mathrm{C}, 1 \mathrm{~h}}{\longrightarrow}$ (b) $\mathrm{Na}_{2} \mathrm{~S}_{2} \mathrm{O}_{4}, \mathrm{H}_{2} \mathrm{O}, 5 \mathrm{~min}$<smiles>O=C1CC(C2=CCCC=C2)Oc2ccc(I)c(O)c21</smiles>
$\left(\mathrm{CH}_{3}\right)_{2} \mathrm{SO}_{4}, \mathrm{~K}_{2} \mathrm{CO}_{3}$ acetone, $25^{\circ} \mathrm{C}, 24 \mathrm{~h}$<smiles>COc1ccc2c(c1OC)C(=O)CC(c1ccccc1)O2</smiles>
DMSO, $90^{\circ} \mathrm{C}, 24 \mathrm{~h}$<smiles>COc1cccc(C(=O)/C=C/c2ccccc2)c1OC</smiles>

图式 13 IBX 参与的黄酮化合物的修饰

Scheme 13 IBX-mediated modification of flavonoids<smiles>[R]C1Cc2cc(OC)c(C)c(OC)c2C(O)O1</smiles>

112<smiles>[R]C1Cc2cc(O)c(C)c(O)c2C=[O+]1</smiles>
113<smiles>[R]C1CC2=CC(=O)[C@@](C)(O)C(=O)C2=CO1</smiles>
d.r. up to $90 / 10$<smiles>[R]C1CC2=CC(=O)[C@@](C)(O[CH+])C(=O)C2=CO1</smiles>

115 single diastereomer

图式 14 二氢吡喃氮酮衍生物的合成

Scheme 14 Synthesis of dihydropyranic azaphilone derivatives 
有的调整碳链长度的简便方法较少, 往往需多步反应才 能实现. 2014 年, Arimoto 等 ${ }^{[50]}$ 在天然产物的全合成研究 过程中, 尝试在乙酸乙酯作溶剂, 回流条件下, 用过量 的 IBX 将醇 116 氧化成醛 117. 但是, 他们并未得到预 期的醛 117 或是其进一步的氧化产物, 而是得到了少一 个碳的羧酸 118. 尽管产率不高, 但这意味着 IBX 可用 于缩短碳链长度(Eq. 30).

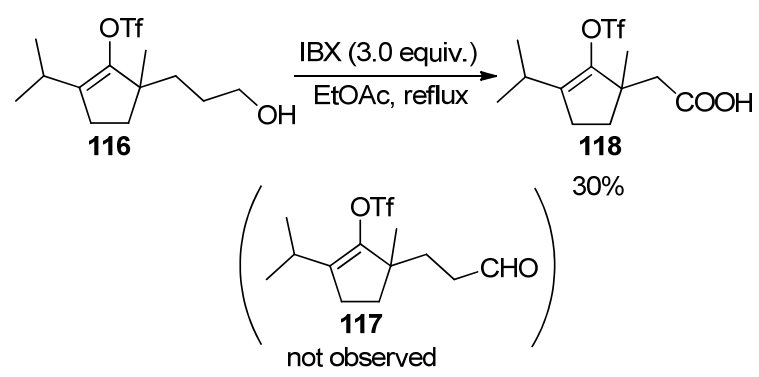

(30)

\subsection{IBX 在形成聚合物中的应用}

2014 年, 李子臣等 ${ }^{[51]}$ 实现了在氧化剂存在下, 以二 羧酸 116、伯醇 117 和二异氰 118 的多组分 Passerini 反 应得到化合物 119. 该反应包括 IBX 氧化伯醇成醛以及 随后的 Passerini 三组分的聚合(Scheme 15). 从醇氧化反 应和 Passerini 反应的反应动力学研究中发现, 这一缩聚 反应十分高效.

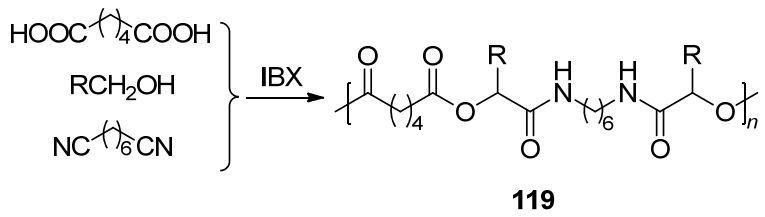

图式 15 三组分聚合

Scheme 15 Three-component polymerization

\subsection{IBX 协同下在羰基化合物的 $\beta$ 位形成碳-碳键}

通过羰基化合物的功能化形成新的 $\mathrm{C}-\mathrm{C}$ 键的研究 在有机合成中具有重大意义 ${ }^{[52]}$. 虽然羰基及其 $\alpha-\mathrm{H}$ 因其 自身的亲电性和酸性，易于在金属试剂参与下形成新的 $\mathrm{C}-\mathrm{C}$ 键. 但要通过活化羰基化合物 $\beta$ 位的 $\mathrm{C}-\mathrm{H}$ 键形成 碳一碳键, 仍具有很大的挑战性. 在 2017 年 $\mathrm{Li}$ 等 ${ }^{[53]}$ 利用 IBX 原位氧化烯醇成 $\alpha, \beta$-不饱和酮, 继而与芳基硼酸发 生钯催化下的偶联反应, 实现了其 $\beta-\mathrm{C}-\mathrm{H}$ 的直接芳基 化反应. 该方法反应可在室温和空气中进行, 水作溶剂, 当采用手性双齿膦配体时，反应的 $e e$ 值可达到 $95 \%$ (Eq. 31).<smiles>O=C1CCCCC1[18OH]</smiles>

120

121

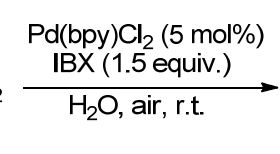

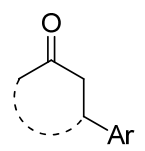

122

\subsection{IBX 在合成 $\alpha$-酮醛中的应用}

$\alpha$-酮醛是合成杂环化合物高活性前体化合物. 2012 年, Schmidt 等 ${ }^{[54]}$ 用异丙基氯化镁和碘仿处理芳醛得到 2,2-二碘芳基乙醇 123, 然后在室温下用 IBX 氧化差基 成崖基，再经二甲亚砜的氧转移作用，原位生成相应的 2-氧代 2-芳基乙醛 124. 124 与双亲核试剂邻苯二胺发生 亲核加成，环合形成稳定的 2-芳基取代喹喔啉化合物 125 (Scheme 16).

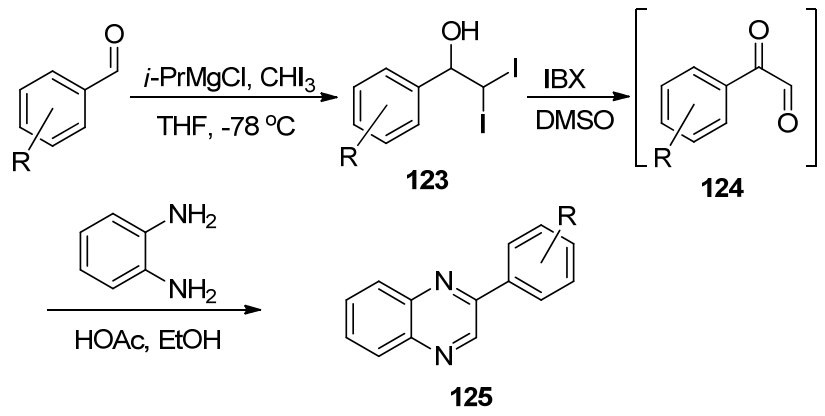

图式 16 IBX 氧化法合成 $\alpha$-酮醛化合物

Scheme 16 Synthesis of $\alpha$-ketone aldehyde via the oxidation by IBX

\subsection{IBX 在有机合成中的其它反应}

2011 年, Akamanchi 等 ${ }^{[55]}$ 发现 IBX 可在氨水中使环 氧化合物 126 发生氨化裂解生成相应的腈 127 (Eq. 32).

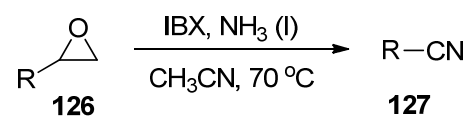

2017 年, Sekar 等 ${ }^{[56]}$ 将仲醇氧化制备 $\alpha$-羟基酮. 他 们利用二甲基亚砜(DMSO)作为溶剂和高价碘离子的配 体，采用 IBX 氧化单质碘原位生成三价碘正离子，继而 将仲醇 128 氧化成酮的同时实现 $\alpha$-差基官能化，从而建 立了制备 $\alpha$-羟基酮 129 化合物的高效方法(Eq. 33).

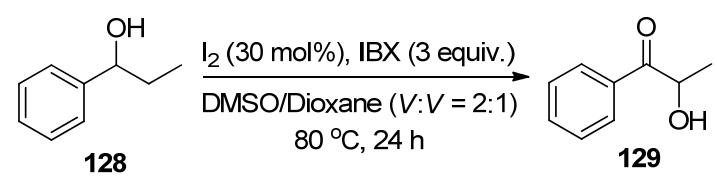

Moorthy 课题组 ${ }^{[57]}$ 多年来一直致力于 IBX 反应性能 的研究. 他们对 IBX 试剂进行了一些改进，并提出了基 于氧化剂过硫酸氢钾制剂(Oxone)氧化 3,4,5,6-四甲基-2碘苯甲酸，原位生成四甲基取代的 IBX 衍生物 TetMeIBX 76 的方法. 这一方法可以让多种烯烃 130 氧化裂 解, 生成相应的酮 132 或酸 131 (Eq. 34) ${ }^{[58]}$. 此外, 当反 应物底物含有两个碳一碳双键时，可利用富电子碳一碳双 键和缺电子碳一碳双键之间反应性的差异，实现对碳一碳 
双键的选择性氧化裂解(Eq. 35).
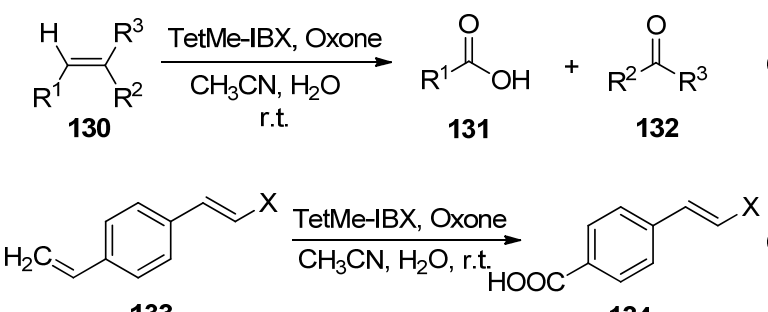

此外, Moorthy 等 ${ }^{[59]}$ 还设计出了一种双 IBX 的结构 139. 139 不仅可将 1,2 -二醇 135 氧化成 $\alpha$-羊基酮 136 (Eq. 36), 还可将 1,4-二醇 137 氧化成内酯化合物 138 (Eq. 37). 使用 TetMe-IBX 也可以实现这一氧化过程 ${ }^{[58]}$. 通 过原位生成的 TetMe-IBX 催化氧化中间产物 $\alpha$-溴代醇 141 (Scheme 17) ${ }^{[61]}$, 可成功实现以烯烃为原料一锅法合 成 $\alpha$-溴代酮 142 和 $\alpha$-叠氮酮 143.<smiles>OCC(O)c1ccc(Br)cc1</smiles>

135

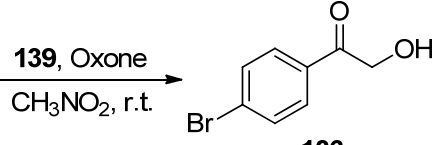

136<smiles>OCc1ccccc1CO</smiles>
137<smiles>Cc1c(C)c(C)c2c(c1C)C(=O)OP2(=O)O</smiles>

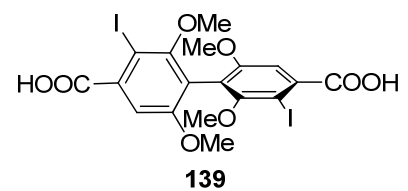

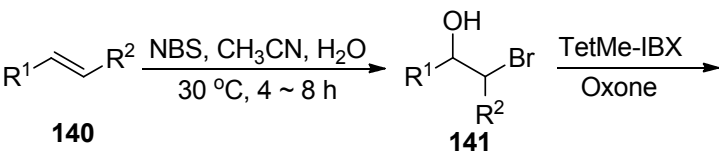



图式 17 TetMe-IBX 催化氧化 $\alpha$-溴代醇

Scheme 17 TetMe-IBX catalytic oxidation of $\alpha$-bromoalkahol

2016 年, Moorthy 等 ${ }^{[62]}$ 用 IBX 将 $\beta$-菜酚氧化成 1,2菜醌, 进一步对氧化产物进行衍生化, 即利用 DielsAlder 环加成和缩合环化反应一锅合成了咔唑吩嗪化合 物 ${ }^{[63]}$, 并用 Wittig 反应和 Michael 加成法一锅合成了苯 并香豆素化合物 ${ }^{[64]}$.

2019 年, Moorthy 等 ${ }^{[65]}$ 用 NIS/IBX 试剂在二甲基亚
砜(DMSO)中，于 $25{ }^{\circ} \mathrm{C}$ 下直接氧化吲哚. 该反应由多取 代的吲哚 144 与 NIS 迅速反应生成中间产物 3-碘代吲哚 145, 再用 IBX 氧化成吲哚酮化合物 146. 由于该反应采 用的是一锅法，故可简便地获取用途广泛的吲哚酮化合 物(Scheme 18).

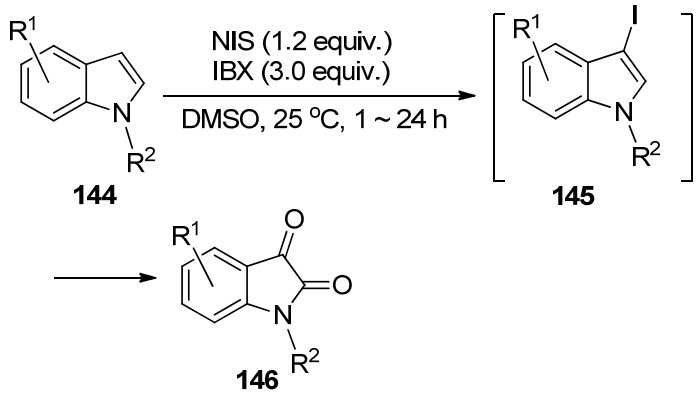

图式 18 IBX 氧化吲哚制备吲哚啉-2,3-二酮

Scheme 18 Synthesis of indoline-2,3-diones via oxidation of indole by IBX

2012 年，刘宏民等 ${ }^{[66]}$ 用 $d$-木糖高效简便地合成茉 莉 B 时, 发现 IBX 是氧化剂中最好的选择. 2018 年, Ito 等 ${ }^{[67]}$ 在胸膜螺旋体的不对称全合成中，也采用了 IBX 作 为氧化剂. 近年来, 也有上百篇专利中, 使用了 IBX 作 为合成中的一环, IBX 正在发挥着越来越大的作用.

\section{IBX 介导的氧化反应的机理及理论研究}

不断有人提出 IBX 介导的氧化反应的可能机理 ${ }^{[68]}$, 其中一种机理认为 $\mathrm{C}-\mathrm{H}$ 键断裂的还原消去是反应的决 速步 [69]. 最近, 关于 IBX 介导的胺和醇的氧化机理的研 究有了新进展. Ariafard 等 ${ }^{[70]}$ 利用密度泛函理论研究了 IBX 氧化胺和醇的可能机理. 他们发现五价高碘化合物 IBX 氧化胺和醇的反应均有着类似的机理. 反应先进行 IBX 的配体交换，得到一个四配位中间体 150, 再进行 氧化还原，最后得到碘(III)化合物和氧化产物 151. 为了 沿着能量上更易进行的路线反应，配体交换和氧化还原 两个步骤均需要进行结构扭曲. 研究发现, 配体的交换 过程需要由水、第二分子 IBX 或者第二分子底物提供质 子. 有趣的是，虽然在配体交换反应中，胺和醇的活化 能几乎相等(特别是由水分子提供质子时), 但在随后的 氧化还原步骤中, 胺的活化能比醇的活化能低很多 (Scheme 19).

$\beta$-硝基苯乙烯是一种重要的有机合成中间体. 它可 作为自由基受体, 形成新的碳-碳键. 2018 年, Akamanchi 等 ${ }^{[71]}$ 报道了 IBX 参与下 $\beta$-硝基苯乙烯与芳基肼的自 由基反应，生成高度立体选择性的 $(E)-1,2$-二苯乙烯化 合物(Eq. 38). 芳基肼 153 在 IBX 的氧化下，脱去氮气， 生成芳基自由基. 芳基自由基与硝基苯乙烯 152 发生自 


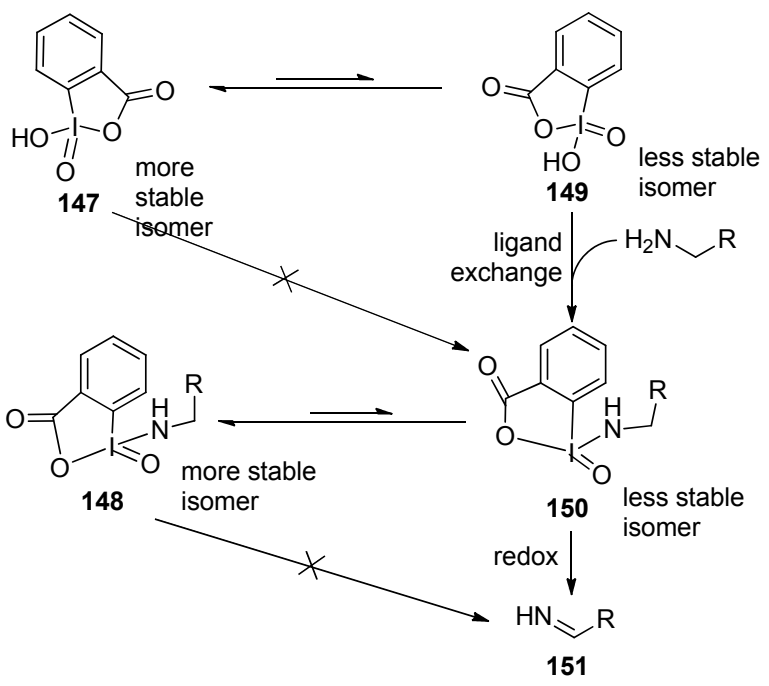

图式 19 IBX 氧化胺和醇的反应机理

Scheme 19 Mechanism of amine and alcohol oxidation mediated by IBX



$\mathrm{R}^{1}=\mathrm{Me}, \mathrm{OMe}, \mathrm{F}, \mathrm{Cl}, \mathrm{Br}, \mathrm{NO}_{2} \quad \mathrm{R}^{2}=\mathrm{H}, 54 \% \sim 80 \%$ yield

$\mathrm{R}^{2}=\mathrm{H}, \mathrm{Me}, \mathrm{OMe}, \mathrm{Cl}, \mathrm{Br} \quad \mathrm{R}^{2}=\mathrm{Me}, \mathrm{OMe}, \mathrm{Cl}, 61 \% \sim 70 \%$ yield

由基加成反应，继而消除硝基自由基，同时形成新的 碳-碳键, 最终得到 $(E)-1,2$-二苯乙烯 154. 该反应可在 敞口的反应器中进行, 反应时间短, 底物适用性广, 无 过渡金属参与, 反应条件温和, 且产物具有高度的 $E$-构 型选择性. (E)-1,3-二甲氧基-5-(4-甲氧基苯乙烯基)苯是 具有抗癌和神经药物的前药化合物, 因而运用这一合成 方法有助于开展以 $(E)$-二苯乙烯为骨架的化合物的抗癌 和神经药物研究.

在药物合成和医学研究方面, IBX 也经常被用作合 成制备中的一环. 近年来在此方面的报道有治疗精神分 裂症部分激动剂的合成 ${ }^{[72]}$ 、酶单活性位点突变 ${ }^{[73]}$ 、医药 稠合杂环的研究 ${ }^{[74]}$ 及微管蛋白聚合抑制剂的研究 ${ }^{[75]}$ 等, 此外, IBX 同样也在抗癌药物的研究 ${ }^{[76]}$ 和合成上发挥着 重要的作用, 在具有良好安全性的新药的合成上具有很 大的应用前景. Wong 团队 ${ }^{[77]}$ 提出了一系列低丰度异冬 青二萜类化合物的合成方法, 并从中得到了一种具有抗 癌活性的候选药物分子.

\section{IBX 试剂的改进}

虽然 IBX 具有诸多优点, 但也存在一些弊端, 其在 高温下易爆炸，在 DMSO 以外的有机溶剂中溶解性差， 这些缺点一度阻碍了 IBX 的应用. 围绕这两个问题, 陆
续出现了关于 IBX 的衍生物及改进操作方法的一些研 究报道.

因 IBX 在高温下容易发生爆炸，使 IBX 只能在低温 环境下使用. 而低温下 IBX 的反应性相对较低, 因而低 温极大限制了 IBX 的应用范围. 为寻找更有效的碘 $(\mathrm{V})$ 氧化剂，Yusubov 等 ${ }^{[78]}$ 合成并研究了 IBX 的对甲苯磺酸 二醋酸衍生物(IBX-OTs diacetate)和双三氟醋酸衍生物 (IBX-ditriflate) 156. 其中, 156 的氧化性最强. 在二氯甲 烷中, 三氟乙酸存在的条件下, IBX 与三氟甲烷磺酸 $(2$ equiv.)反应生成 IBX 的双三氟醋酸衍生物 156 (Eq. 39).

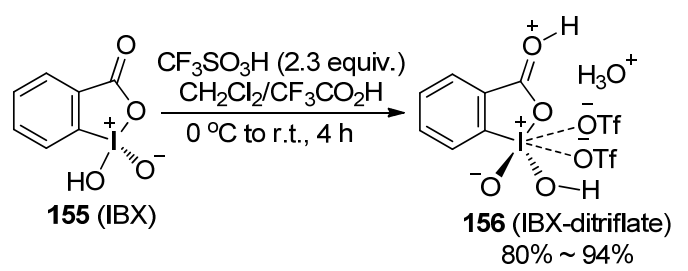

2011 年, Pivnitsky 等 ${ }^{[79]}$ 提出稳定易得的 IBX 的吡啶 盐 158 (PIBX) 可作为 IBX 氧化剂的更安全的替代品. 他 们发现 PIBX 在极性溶剂[如 $N, N$-二甲基甲酰胺(DMF) 和 DMSO]中, 在将醇氧化成酮或醛的过程中表现出与 IBX 相当的反应性, 并且因为 PIBX 在溶剂中的溶解性 更好, 可在四氢呋喃(THF)中达到更高的氧化速率(Eq. 40). 用 PIBX在 DMF 中, 室温条件下将甾醇氧化成甾酮 的产率高达 97\%.

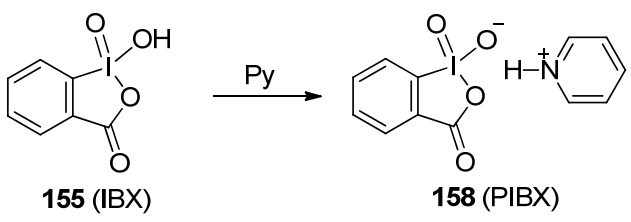

2014 年, $\mathrm{Mal}$ 等 ${ }^{[80]}$ 没有遵循传统的依靠改造 IBX 分 子结构的或阳离子以改善 IBX 易爆炸、反应性低和溶解 度差的缺点, 提出了另一种解决问题的思路. 他们利用 一个直径为 $15 \mathrm{~mm}$ 的氧化锆球体, 在无溶剂条件下依靠 氧化锆球磨法使 IBX 在室温下可有效发挥其氧化作用, 且此法对 IBX 的传统氧化反应均能适用. 例如, 将球磨 法应用于将伯醇和仲醇氧化成相应的羰基化合物，将胺 氧化成亚胺, 将硫醚氧化成亚砜, 将烯键氧化成 $\alpha$-溴代 酮或 $\alpha$-碘代酮等反应均能得到较好的反应结果. 更重要 的是, IBX 的还原产物 2-碘代苯甲酸(IBA) 可以在反应过 程中原位再生成 IBX. 实验表明, IBX 经过 15 次循环使 用后其反应活性仅下降了 $6 \%$, 大大提高了 IBX 的使用 效率. 而且, 这一方法具有较宽的量级范围 $(10 \mathrm{mg} \sim 2.5$ g). 因而这一有机小分子的机械化学合成方法 ${ }^{[81]}$ 成功解 决了 IBX 在克量级和非爆炸性条件下使用效率较低的 问题. 
为克服 IBX 不溶于常见的有机溶剂这一缺点, Yakura 等 ${ }^{[82]}$ 制备了取代的 2-碘代苯甲酸、2-碘代苯磺酸、 $N$-异丙基-2-碘代苯甲酰胺和 4-碘代苯磺酸等化合物, 研究了这些化合物在过硫酸氢钾制剂(Oxone)和四丁基 硫酸氢铵存在下将醇氧化成酮的反应. 结果发现, 在硝 基甲烷/水溶剂中取代的 $N$-异丙基-2-碘代-苯甲酰胺化 合物的反应活性最高, 反应产率可达 $99 \%$. 此外, 近年 来关于 IBX 的修饰, 以及能与水共溶的 IBX 衍生物 ${ }^{[83]}$ 、 更稳定的 IBX 衍生物、反应性或选择性更高的 IBX 衍 生物的合成研究均有大量报道.

\section{0 结语}

综上所述, 近 20 年来, 高价碘试剂凭借自身独特的 反应性 以及与过渡金属相似的性质和低毒性引起了众 多有机化学家的关注. 在已经报道的高价碘试剂中, 2碘酰基苯甲酸(IBX)因具有能溶于 DMSO, 反应条件温 和、选择性好、产率高、环境友好、易于制备、价格低 廉及可长期保存等优点而备受关注. IBX 目前已在氧化 差基、含氮化合物、含硫化合物以及制备 $\alpha, \beta$-不饱和羰 基化合物、酯和不对称合成等方面获得广泛应用. 此外, IBX 工业制备方法的出现，也为其在工业领域中的应用 打下坚实基础. 尽管 IBX 仍存在高温下易爆炸和在 DMSO 以外的普通有机溶剂中溶解性差这两大缺点, 但 随着对 IBX 分子结构和应用条件的不断改进和探索, 将 使 IBX 的应用的范围和前景进一步扩大.

\section{References}

[1] Willgerodt, C. J. Prakt. Chem. 1886, 33, 154.

[2] Dess, D. B.; Martin, J. C. J. Org. Chem. 1983, 48, 4155.

[3] (a) Reddy Kandimalla, S.; Prathima Parvathaneni, S.; Sabitha, G.; Subba Reddy, B. V. Eur. J. Org. Chem. 2019, 2019, 1687.

(b) Zhang, H.; Tang, R.; Shi, X.; Xie, L; Wu, J. W. Chin. J. Org. Chem. 2019, 39, 1837 (in Chinese).

(张怀远, 唐蓉萍; 石星丽; 颉林; 伍家卫, 有机化学, 2019, 39, 1837.)

(c) Liu, D.; He, J.; Zhang, C. Univ. Chem. 2019, 34, 1 (in Chinese). (刘丹, 贺家豪, 张弛, 大学化学, 2019, 34, 1).

(d) Li, X.; Chen, P.; Liu, G. Beilstein J. Org. Chem. 2018, 14, 1813. (e) Gao, H.; Huang, J.; Jiang, Q.; Guo, W.; Ge, C.; Jiang, H. Chem. Reagents 2016, 38, 1165 (in Chinese).

(高浩凌, 黄骏, 江群, 郭文光, 葛承胜, 姜洪涛, 化学试剂, 2016, 38, 1165.)

(f) Duan, Y.-N.; Jiang, S.; Han, Y.-C.; Sun, B.; Zhang, C. Chin. J. Org. Chem. 2016, 36, 1973 (in Chinese)

(段亚南，姜山，韩永超，孙博，张弛，有机化学, 2016, 36, 1973.) (g) Chen, J.; Qu, H.; Peng, J.; Chen, C. Chin. J. Org. Chem. 2015, 35, 937 (in Chinese).

(陈静，曲红梅，彭静，陈超，有机化学, 2015, 35, 937.)

[4] (a) Satam, V.; Harad, A.; Rajule, R.; Pati, H. Tetrahedron 2010, 66, 7659 .

(b) Qin, K. Y.; Su, G. F.; Rao, W. P.; Tan, G. M. Chin. J. Org. Chem. 2006, 26, 1623 (in Chinese).

(覃开云, 苏桂发, 饶万平, 谭光明, 有机化学, 2006, 26, 1623.)

[5] Frigerio, M.; Santagostino, M. Tetrahedron Lett. 1994, 35, 8019.

[6] Wang, Y.-H.; Cong, H.; Zhao, F.-F.; Xue, S.-F.; Tao, Z.; Zhu, Q.-J.;
Wei, G. Catal. Commun. 2011, 12, 1127.

[7] Bartlett, S. L.; Beaudry, C. M. J. Org. Chem. 2011, 76, 9852.

[8] Liu, Y.; Xie, A.; Cao, M.; Feng, L.; Wang, B. Asian J. Chem. 2015 $27,587$.

[9] Xie, A.; Zhou, X.; Feng, L.; Hu, X.; Dong, W. Tetrahedron 2014, $70,3514$.

[10] Kumar, S.; Ahmed, N. Green Chem. 2016, 18, 648.

[11] Raghavan, S.; Kumar, V. V. Tetrahedron 2013, 69, 4835.

[12] Kamal, A.; Shaik, A. B.; Jain, N.; Kishor, C.; Nagabhushana, A.; Supriya, B.; Bharath Kumar, G.; Chourasiya, S. S.; Suresh, Y.; Mishra, R. K.; Addlagatta, A. Eur. J. Med. Chem. 2015, 92, 501.

[13] Kinoshita, Y.; Kitagawa, Y.; Tamiaki, H. Chem.-Eur. J. 2016, 22, 9996.

[14] Kawase, M.; Saijo, R.; Mori, S.; Uno, H. Heterocycles 2017, 94, 2103.

[15] Karimov, R. R.; Tan, D. S.; Gin, D. Y. Tetrahedron 2018, 74, 3370.

[16] Parasuraman, K.; Chennaiah, A.; Dubbu, S.; Ibrahim Sheriff, A. K.; Vankar, Y. D. Carbohydr. Res. 2019, 477, 26.

[17] (a) Dai, D.; Venepalli, B. R. Tetrahedron Lett. 2015, 56, 2402. (b) Stephenson, G. R.; Roe, C.; Anson, C. E. J. Org. Chem. 2012, $77,9684$.

[18] Lu, T.-J.; Lin, C.-K. J. Org. Chem. 2011, 76, 1621.

[19] Takale, B. S.; Telvekar, V. N. Chem. Lett. 2010, 39, 546

[20] Chen, L.-X.; Huang, Y.-H.; Cong, H.; Tao, Z. Chem. Pap. 2018, 72, 661.

[21] Narayana Murthy, S.; Nageswar, Y. V. D. Tetrahedron Lett. 2011, 52,4481 .

[22] Chandrasekar, S.; Sekar, G. Org. Biomol. Chem. 2016, 14, 3053.

[23] Parmeggiani, C.; Matassini, C.; Cardona, F.; Goti, A. Synthesis 2017, 49, 2890.

[24] Matassini, C.; Parmeggiani, C.; Cardona, F.; Goti, A. Org. Lett. 2015, 17, 4082 .

[25] Moorthy, J. N.; Neogi, I. Tetrahedron Lett. 2011, 52, 3868.

[26] Alam, M. T.; Maiti, S.; Mal, P. Beilstein J. Org. Chem. 2018, 14 , 2396.

[27] Khan, P. R.; Durgaprasad, M.; Reddy, S. G.; Reddy, G. R.; Hussein, I. A.; Subba Reddy, B. V. Lett. Org. Chem. 2018, 15, 64

[28] Wagh, Y. S.; Tiwari, N. J.; Bhanage, B. M. Tetrahedron Lett. 2013 $54,1290$.

[29] Victor, N. J.; Muraleedharan, K. M. Adv. Synth. Catal. 2014, 356, 3600 .

[30] Makra, Z.; Puskas, L. G.; Kanizsai, I. Org. Biomol. Chem. 2019, 17, 9001.

[31] Harschneck, T.; Hummel, S.; Kirsch, S. F.; Klahn, P. Chem.-Eur. J. 2012, 18, 1187.

[32] Potturi, H. K.; Gurung, R. K.; Hou, Y. J. Org. Chem. 2012, 77, 626.

[33] Zhang, Z.; Zheng, D.; Wan, Y.; Zhang, G.; Bi, J.; Liu, Q.; Liu, T.; Shi, L. J. Org. Chem. 2018, 83, 1369.

[34] Ambule, M. D.; Tripathi, S.; Ghoshal, A.; Srivastava, A. K. Chem. Commun. 2019, 55, 10872.

[35] Zhang, Z. G.; Li, X.; Song, M. M.; Wang, Y. M.; Zheng, D.; Zhang, G. S.; Chen, G. J. Org. Chem. 2019, 84, 12792.

[36] Shukla, V. G.; Salgaonkar, P. D.; Akamanchi, K. G. J. Org. Chem. 2003, 68, 5422 .

[37] Poeira, D. L.; Macara, J.; Faustino, H.; Coelho, J. A. S.; Gois, P. M. P.; Marques, M. M. B. Eur. J. Org. Chem. 2019, 2019, 2695.

[38] Moorthy, J. N.; Senapati, K.; Parida, K. N.; Jhulki, S.; Sooraj, K.; Nair, N. N. J. Org. Chem. 2011, 76, 9593.

[39] Ogawa, S.; Zhou, B.; Kimoto, Y.; Omura, K.; Kobayashi, A.; Higashi, T.; Mitamura, K.; Ikegawa, S.; Hagey, L. R.; Hofmann, A. F.; Iida, T. Steroids 2013, 78, 927

[40] Ren, J.; Lu, L.; Xu, J.; Yu, T.; Zeng, B.-B. Synthesis 2015, 47, 2270

[41] Kuga, T.; Sasano, Y.; Iwabuchi, Y. Chem. Commun. 2018, 54, 798.

[42] Chaves, M. R. B.; Moran, P. J. S.; Rodrigues, J. A. R. J. Mol. Catal. B: Enzym. 2013, 98, 73.

[43] Satish, G.; Polu, A.; Ramar, T.; Ilangovan, A. J. Org. Chem. 2015, $80,5167$.

[44] Sundaravelu, N.; Chakraborty, A.; Sekar, G. ChemistrySelect 2018 , 
3,8167 .

[45] Saidhareddy, P.; Ajay, S.; Shaw, A. K. Tetrahedron 2017, 73, 4407.

[46] Kang, Y. K.; Kim, D. Y. Chem. Commun. 2014, 50, 222.

[47] Barontini, M.; Bernini, R.; Crisante, F.; Fabrizi, G. Tetrahedron 2010, 66, 6047 .

[48] Desai, V. G.; Desai, S. R. Curr. Org. Synth. 2017, 14, 1180.

[49] Boulange, A.; Peixoto, P. A.; Franck, X. Chem.-Eur. J. 2011, 17, 10241.

[50] Xu, S.; Itto, K.; Satoh, M.; Arimoto, H. Chem. Commun. 2014, 50, 2758.

[51] Kan, X.-W.; Deng, X.-X.; Du, F.-S.; Li, Z.-C. Macromol. Chem. Phys. 2014, 215, 2221.

[52] Mukherjee, S.; Yang, J. W.; Hoffmann, S.; List, B. Chem. Rev. 2007, 107, 5471.

[53] Hu, X.; Yang, X.; Dai, X.-J.; Li, C.-J. Adv. Synth. Catal. 2017, 359, 2402.

[54] Zall, A.; Bensinger, D.; Schmidt, B. Eur. J. Org. Chem. 2012, 2012, 1439.

[55] Deshmukh, S. S.; Huddar, S. N.; Jadhav, R. R.; Akamanchi, K. G. Tetrahedron Lett. 2011, 52, 4533.

[56] Guha, S.; Kazi, I.; Mukherjee, P.; Sekar, G. Chem. Commun. 2017, $53,10942$.

[57] Mishra, A. K.; Moorthy, J. N. Org. Chem. Front. 2017, 4, 343.

[58] Moorthy, J. N.; Parida, K. N. J. Org. Chem. 2014, 79, 11431.

[59] Seth, S.; Jhulki, S.; Moorthy, J. N. Eur. J. Org. Chem. 2013, 2013, 2445.

[60] Jhulki, S.; Seth, S.; Mondal, M.; Moorthy, J. N. Tetrahedron 2014, 70, 2286.

[61] Chandra, A.; Parida, K. N.; Moorthy, J. N. Tetrahedron 2017, 73, 5827.

[62] Mishra, A. K.; Moorthy, J. N. J. Org. Chem. 2016, 81, 6472.

[63] Mishra, A. K.; Mukhopadhyay, A.; Moorthy, J. N. Tetrahedron 2017, 73, 2210.

[64] Chandra, A.; Jana, K.; Moorthy, J. N. ACS Omega 2020, 5, 207.

[65] Chandra, A.; Yadav, N. R.; Moorthy, J. N. Tetrahedron 2019, 75, 2169.

[66] Zhao, M.-L.; Zhang, E.; Gao, J.; Zhang, Z.; Zhao, Y.-T.; Qu, W.; Liu, H.-M. Carbohydr. Res. 2012, 351, 126.
[67] Kobayashi, T.; Tanaka, K.; Ishida, M.; Yamakita, N.; Abe, H.; Ito, H. Chem. Commun. 2018, 54, 10316.

[68] Tognetti, V.; Boulangé, A.; Peixoto, P. A.; Franck, X.; Joubert, L. J. Mol. Model. 2014, 20, 2342.

[69] Jiang, H.; Sun, T.-Y.; Wang, X.; Xie, Y.; Zhang, X.; Wu, Y.-D.; Schaefer, H. F. Org. Lett. 2017, 19, 6502.

[70] Chipman, A.; Farshadfar, K.; Smith, J. A.; Yates, B. F.; Ariafard, A. J. Org. Chem. 2020, 85, 515.

[71] Wagh, G.; Autade, S.; Patil, P. C.; Akamanchi, K. G. New J. Chem. 2018, 42, 3301.

[72] Magano, J.; Acciacca, A.; Akin, A.; Collman, B. M.; Conway, B.; Waldo, M.; Chen, M. H.; Mennen, K. E. Org. Process Res. Dev. 2009, 13, 555 .

[73] Koch, A. A.; Hansen, D. A.; Shende, V. V.; Furan, L. R.; Houk, K. N.; Jimenez-Oses, G.; Sherman, D. H. J. Am. Chem. Soc. 2017, 139, 13456.

[74] Singh, A.; Mir, N. A.; Choudhary, S.; Singh, D.; Sharma, P.; Kant, R.; Kumar, I. RSC Adv. 2018, 8, 15448 .

[75] Mullagiri, K.; Nayak, V. L.; Sunkari, S.; Mani, G. S.; Guggilapu, S. D.; Nagaraju, B.; Alarifi, A.; Kamal, A. MedChemComm 2018, 9, 275.

[76] Srinivas, A.; Karthik, P.; Sunitha, M.; Reddy, K. V. Acta Chim. Slovaca 2019, 66, 700 .

[77] Zhu, L.; Ma, W.; Zhang, M.; Lee, M. M.-L.; Wong, W.-Y.; Chan, B. D.; Yang, Q.; Wong, W.-T.; Tai, W. C.-S.; Lee, C.-S. Nat. Commun. 2018, 9,1 .

[78] Yusubov, M. S.; Soldatova, N. S.; Postnikov, P. S.; Valiev, R. R.; Yoshimura, A.; Wirth, T.; Nemykin, V. N.; Zhdankin, V. V. Chem. Commun. 2019, 55, 7760.

[79] Kumanyaev, I. M.; Lapitskaya, M. A.; Vasiljeva, L. L.; Pivnitsky, K. K. Mendeleev Commun. 2012, 22, 129.

[80] Achar, T. K.; Maiti, S.; Mal, P. RSC Adv. 2014, 4, 12834.

[81] Achar, T. K.; Bose, A.; Mal, P. Beilstein J. Org. Chem. 2017, 13, 1907.

[82] Yakura, T.; Fujiwara, T.; Yamada, A.; Nambu, H. Beilstein J. Org. Chem. 2018, 14, 971

[83] Cui, L.-Q.; Dong, Z.-L.; Liu, K.; Zhang, C. Org. Lett. 2011, 13, 6488.

(Zhao, C.) 